\title{
Conhecimentos dos estudantes de medicina sobre oncologia: Estudo com internos
}

\author{
Knowlegde of medical students about oncology: Study with interns \\ Conocimiento de estudiantes médicos sobre oncología: Estudio con interno
}

Recebido: 16/06/2021 | Revisado: 25/06/2021 | Aceito: 27/06/2021 | Publicado: 11/07/2021

\author{
Victor César Urquiza Candeia \\ ORCID: https://orcid.org/0000-0003-4399-1185 \\ Centro Universitário de Patos, Brasil \\ E-mail: victorcandeia@med.fiponline.edu.br \\ Milena Nunes Alves de Sousa \\ ORCID: https://orcid.org/0000-0002-9431-6326 \\ Centro Universitário de Patos, Brasil \\ E-mail: milenanunes@fiponline.edu.br
}

\begin{abstract}
Resumo
Objetivou-se avaliar o conhecimento de estudantes de medicina sobre o rastreio precoce, a dor e o manejo dos cuidados paliativos na oncologia. Foi adotado estudo transversal, descritivo, com abordagem quantitativa. Participaram 101 internos do curso de medicina do Centro Universitário de Patos, Paraíba. Para a coleta de dados, realizada entre fevereiro e abril de 2021, utilizou-se um questionário adaptado, disponibilizado virtualmente mediante Google Forms. Os dados foram analisados no software Statistical Package for the Social Sciences (SPSS), versão 25. Foram utilizadas estatísticas descritivas das variáveis demográficas e medidas de tendência central. As respostas envolveram uma escala tipo Likert, variando de 0 (discordo totalmente) a 4 (concordo totalmente). Para fazer a comparação das médias do conhecimento e segurança em Oncologia, foi primeiro realizado o Teste de Normalidade de Shapiro-Wilk e o teste de Mann-Whitney para a comparação de médias entre grupos do $5^{\circ}$ e $6^{\circ}$ ano. Adotou-se significância estatística de $\mathrm{p} \leq 0,05$. Por fim, foi reconhecido que, embora os acadêmicos possuam um nível de conhecimento aceitável, é necessário aprimorar a oportunidade de oferta desse conhecimento, haja vista a importância e a prevalência epidemiológica desse agravo. Sendo assim fica nítida a necessidade de um maior enfoque dessa temática na formação acadêmica e nas produções científicas desses futuros médicos.
\end{abstract}

Palavras-chave: Estudantes de medicina; Oncologia; Conhecimento; Ensino.

\begin{abstract}
The aim was to assess the knowledge of medical students about early detection, pain and palliative care management in oncology. A cross-sectional, descriptive with a quantitative approach study was adopted. A hundred and one medical interns of the medicine course of Centro Universitário de Patos, Paraíba. To collect data, between february and april of 2021, it was used an adapted questionnaire, available virtually through Google Forms. The data were analyzed on the Statistical Package of the Social Sciences (SPSS), 25 version. descriptive statistics were used on the demographics variables and central tendency measures. The answers involved a Likert-type scale, ranging from 0 (strongly disagree) to 4 (strongly agree). To make the comparison of means of knowledge and reliability in oncology, it was used the Shapiro-Wilk normality test and the Mann-Whitney test to compare the means between the 5th and 6th year groups of interns. The statistical significance adopted was 5\%. Lastly, it was recognized that, although academics have an acceptable level of knowledge, it is necessary to improve the opportunity to offer this knowledge, given the importance and epidemiological prevalence of this disease. Therefore, there is a clear need for a greater focus on this theme in academic training and in the scientific production of these future physicians.
\end{abstract}

Keywords: Medical students; Oncology; Knowledge; Teaching.

\section{Resumen}

Este estudio tuvo como objetivo evaluar el conocimiento de los estudiantes de medicina sobre el cribado precoz, el dolor y el manejo de los cuidados paliativos en oncología. Se adoptó un estudio descriptivo transversal con enfoque cuantitativo. Participaron 101 internos del curso de medicina del Centro Universitario de Patos, Paraíba. Para la recogida de datos, realizada entre febrero y abril de 2021, se utilizó un cuestionario adaptado, disponible virtualmente a través de Google Forms. Los datos se analizaron mediante el software Statistical Package for Social Sciences (SPSS), versión 25. Se utilizó estadística descriptiva de variables demográficas y medidas de tendencia central. Las respuestas incluían una escala tipo Likert, que iba de 0 (muy en desacuerdo) a 4 (muy de acuerdo). Para comparar las medias de conocimiento y seguridad en Oncología, primero se realizaron la prueba de normalidad de Shapiro-Wilk y la prueba de Mann-Whitney para comparar medias entre los grupos de $5^{\circ}$ y $6^{\circ}$ año. Se adoptó una significación estadística de $\mathrm{p}<0,05$. Finalmente, se reconoció que, aunque los académicos tienen un nivel de conocimiento aceptable, es necesario mejorar la oportunidad de ofrecer este conocimiento, dada la importancia y prevalencia 
epidemiológica de este agravio. Por tanto, existe una clara necesidad de un mayor enfoque de este tema en la formación académica y en la producción científica de estos futuros médicos.

Palabras clave: Estudiantes de medicina; Oncología; Conocimiento; Ensenãnza.

\section{Introdução}

Por definição, o câncer é entendido como um grupo com diversas doenças caracterizadas pela multiplicação e pelo crescimento descontrolado que possuem a capacidade de espalhar-se entre os tecidos e órgãos próximos à estrutura acometida inicialmente (Batista, Mattos \& Silva, 2015). O câncer é considerado grande problema de saúde pública, devido a sua dimensão epidemiológica, social e econômica (Santos et al., 2021).

Ao pensar em saúde pública, não há dúvidas que o câncer representa uma grande dificuldade, sobretudo entre os países em desenvolvimento. Espera-se que, dentre os mais de 20 milhões de novos casos previstos para 2025, 80\% ocorram nessas nações. Outro estudo feito em 2012, realizado em escala mundial, constatou-se que dos 14 milhões dos novos casos, mais de 60\% aconteceram em países em desenvolvimento (Theobald, Santos, Andrade \& De-Carli, 2016).

A incidência crescente de casos de neoplasia tem ocasionado uma transformação no perfil epidemiológico da população, seja pelo aumento da exposição aos fatores cancerígenos, pelo envelhecimento populacional, pelo aprimoramento das tecnologias para o diagnóstico, como também pela elevação do número de óbitos por câncer (Batista, Mattos \& Silva, 2015; Silva et al., 2021). No Brasil, tem sido um fator responsável pela sobreposição das doenças crônico-degenerativas sobre as afecções agudas, com ênfase para o câncer (Dantas et al., 2020).

Obter um diagnóstico de câncer gera consequências multifatoriais na vida do paciente e de seus amigos e familiares, que vão desde sentimentos de insegurança e medo, ansiedades, vulnerabilidade, até alterações em seus projetos de vida pessoais e profissionais causadas pelo adoecimento (Batista, Mattos \& Silva, 2015; Silva, Silva \& Porfírio, 2020).

Ao considerar o contexto e ao compreender-se que a detecção precoce do câncer é um parâmetro capaz de diminuir o índice de mortalidade dos pacientes oncológicos. Além disso, constata-se que a eficácia dos médodos de rastreamento é multifatorial, mas que a função do médico é relevante ao sucesso da prática. Entretanto, pesquisa atual evidenciou carência no conhecimento médico sobre tal temática (Silvestrini, Scherrer \& Moreira, 2012). Ainda para os autores, o fato de os alunos reconhecerem a falta de conhecimento médico como barreira ao rastreio adequado reforça a importância do ensino adequado do componente curricular nas universidades.

É imprescindível que haja uma assistência qualificada e efetiva por parte dos profissionais de saúde envolvidos no tratamento do câncer. Sendo assim, devido à alta complexidade e individualização dos sintomas dessa patologia, é necessária uma capacitação constante dos profissionais de saúde. Porém, constata-se um déficit evidente na preparação desses profissionais, sobretudo na graduação, visto que muitos desses cursos de saúde não oferecem o aprofundamento necessário nessa área (Luz, Vargas, Rosa, \& Schmitt, 2016).

Estudo realizado com pacientes diagnosticados com câncer, em três serviços de oncologia clínica associados a uma Faculdade de Medicina do ABC, no interior do Estado de São Paulo, foi observada uma falha no processo de diagnóstico precoce e início imediato do tratamento, fatores que podem configurar em um mal prognóstico e reduzida chance de cura dessa patologia (Batista, Mattos \& Silva, 2015).

A redução da eficácia do diagnóstico precoce e do tratamento ofertado ao paciente onconcológico leva a consequências irreversíveis, o início do sofrimento e a antecipação da morte passam a ser algo esperado, embora sejam feitos naturais da vida (Kameo et al., 2021). Paradoxalmente, observa-se que o modelo preponderante de ensino e de prática da Medicina não oferece a merecida ênfase a tais temas e constata-se um desapreço crescente dos profissionais médicos para tratar dessas temáticas (Pinheiro, 2010; Peirano et al., 2018).

Com o diagnóstico do câncer em estágios mais avançados, o tratamento em tempo integral se torna cada vez mais 
necessário. Dessa forma, os cuidados paliativos (CP) são ações ofertadas por uma equipe multidiciplinar de saúde com a finalidade de aliviar os sintomas desagraveis e até incapacitantes, além de promover uma redução dos gastos hospitalares. Em fases mais avançadas da doença, o atendimento humanizado somado a CP, vincula-se com resultados cada vez melhores e com finais de vida menos agressivos (Peirano et al., 2018; Silveira, Costa, Lohmann \& Lavall (2020).

Os CP configuram um modelo de cuidado onde o objetivo do tratamento é deslocado da patologia para o paciente em sua integridade, que vai desde as suas raizes de vida e a sua realidade familiar e cultural, até o seu processo de adoecimento e de morte, levando certo conforto biopsicosocial para todos (Fonseca \& Geovanini, 2013; Silva et al., 2021).

Dessa forma, o profissional capacitado em exercer os CP deve possuir, dentre outras, as habilidades, o trabalho em equipe, a excelência quanto ao manejo da doença em seu estado mais avançado, das medicações específicas para sanar e/ou reduzir a dor e o desconforto, além de médotos de apoio e de enfrentamento do processo de luto e da morte, que os amigos, familiriares e profissionais experimentam (Fonseca \& Geovanini, 2013). Ademais, uma das maiores dificuldades do acesso dos pacientes oncológicos aos cuidados paliativos é o baixo conhecimento das equipes com relação à necessidade dos pacientes (Peirano et al., 2018; Silveira, Costa, Lohmann \& Lavall (2020); Silva et al., 2021).

Diante o exposto e ao considerar a experiência dos pesquisadores deste estudo, tem-se observado a importância da obtenção do conhecimento, por parte dos estudantes de medicina, sobre o diagnóstico precoce, a dor e o manejo dos cuidados paliativos nos pacientes oncológicos, acarretando além de um melhor prognóstico e uma melhoria no atendimento ao paciente, uma maior segurança no atendimento a um paciente oncológico. Propõe-se, assim, avaliar o conhecimento de estudantes de medicina sobre o rastreio precoce, a dor e o manejo dos cuidados paliativos na oncologia.

\section{Metodologia}

Trata-se de um estudo descritivo, transversal com abordagem quantitativa, que consiste em uma pesquisa na qual a coleta dos dados quantitativos ou numéricos é obtida do emprego de medições de grandezas e obtém-se por meio da metrologia, número com suas respectivas unidades. Estas técnicas geram conjuntos ou massas de dados com suas que podem ser examinados por meio de técnicas matemáticas como os métodos analíticos, estatísticas e probabilidades, geração de equações, entre outros (Soares, Shitsuka, Parreira \& Shitsuka, 2018).

O presente estudo avaliou uma amostra não probabilística por conveniência. Participaram 101 internos (69,18\% da população total) do $5^{\circ}$ e $6^{\circ}$ ano, que estavam no Internato ou Estágio Curricular Obrigatório de Formação em Serviço (ECOFS) do curso de medicina do Centro Universitário de Patos (UNIFIP), localizado em Patos, Paraíba. No primeiro ano de internato, cumprem os estágios em Medicina de Família e Comunidade, Saúde Mental, Clínica Cirúrgica, Ginecologia e Obstetrícia; no segundo ano em Pediatria, Clínica Médica, Urgência e Emergência. Excluíram-se os estudantes não matriculados no período de coleta de dados, os não blocados no ECOFS e os participantes com o questionário incompleto.

Após prévia autorização do Comitê de Ética, CAAE 33436920.5.0000.5181 sob Protocolo de nº 4.423.561/2020. A coleta de dados procedeu-se a partir da ferramenta Google Forms e o questionário conteve 20 questões fechadas. Vale ressaltar que as questões usadas foram adaptadas de outros estudos (Pinheiro, 2010; Frizzo, Bertolini, Caron, Steffani, \& Bonamigo, 2013; Orth et al., 2019; Sousa \& Roriz, 2021). O período de coleta compreendeu os meses de fevereiro a abril do ano de 2021. Antes de dar início à participação na pesquisa, os internos assinaram o Termo de Consentimento Livre e Esclarecido (TCLE), disponibilizado também de forma online.

Os dados foram analisados por meio do software Statistical Package for the Social Sciences (SPSS), versão 25. Foram utilizadas estatísticas descritivas das variáveis demográficas da amostra e medidas de tendência central para as variáveis resultantes dos itens do questionário, que contemplavam a variável "Conhecimento" e "Oportunidade de Conhecimento". As respostas envolveram uma escala tipo Likert, variando de 0 (discordo totalmente) a 4 (concordo totalmente). 
Para fazer a comparação das médias do conhecimento e segurança em Oncologia, foi primeiro realizado o Teste de Normalidade de Shapiro-Wilk para conhecer a natureza do teste que irá ser aplicado (paramétrico ou não paramétrico), significância estatística de $\mathrm{p} \leq 0,05$. Sendo assim, utilizou-se o teste de Mann-Whitney para a comparação de médias entre grupos do $5^{\circ}$ e $6^{\circ}$ ano de Medicina.

\section{Resultados}

Em conformidade com o Quadro 1, foi visto que a maior parte dos estudantes possuía entre 23-26 anos (53,5\%; $\mathrm{N}=54)$, era do sexo feminino $(60,4 \% ; \mathrm{N}=61)$, estado civil solteiro $(97 \% ; \mathrm{N}=98)$ e com relação à ocupação, foi visto que $89,1 \%$ ( $\mathrm{N}=90)$ apenas estudava. Quanto ao estágio curricular, foi analisado que a maioria estava no rodízio de APS (30,7\%; N=31), e que $67,33 \%(\mathrm{~N}=68)$ eram do $5^{\circ}$ ano do curso de medicina.

Quadro 1: Frequências absolutas e relativas das variáveis Idade, Sexo, Estado Civil, Ocupação além dos Estudos e Rodízio do Internato.

\begin{tabular}{|c|c|c|}
\hline Sexo & Frequência & Percentual \\
\hline Masculino & 40 & 39,6 \\
\hline Feminino & 61 & 60,4 \\
\hline Total & 101 & 100,0 \\
\hline Faixa etária & Frequência & Percentual \\
\hline $18-22$ & 27 & 26,7 \\
\hline $23-26$ & 54 & 53,5 \\
\hline $27-30$ & 16 & 15,8 \\
\hline $30+$ & 4 & 4,0 \\
\hline Total & 101 & 100,0 \\
\hline Estado Civil & Frequência & Porcentual \\
\hline Solteiro & 98 & 97,0 \\
\hline Casado & 3 & 3,0 \\
\hline Total & 101 & 100,0 \\
\hline Ocupação além dos estudos & Frequência & Porcentual \\
\hline Apenas Estudo & 90 & 89,1 \\
\hline Estudo e trabalho & 11 & 10,9 \\
\hline Total & 101 & 100,0 \\
\hline Rodízio & Frequência & Porcentual \\
\hline APS & 31 & 30,7 \\
\hline Saúde Mental & 1 & 1,0 \\
\hline Ginecologia e Obstetrícia & 25 & 24,8 \\
\hline Cirurgia & 11 & 10,9 \\
\hline Pediatria & 15 & 14,9 \\
\hline UeE & 14 & 13,9 \\
\hline Clínica Médica & 4 & 4,0 \\
\hline Total & 101 & 100,0 \\
\hline $\begin{array}{c}\text { Ano de estudo em curso na } \\
\text { Medicina }\end{array}$ & Frequência & Percentual \\
\hline $5^{\circ}$ Ano & 68 & 67,33 \\
\hline $6^{\circ}$ Ano & 33 & 32,67 \\
\hline Total & 101 & 100,0 \\
\hline
\end{tabular}

Fonte: Dados da pesquisa (2021).

Foi construída a variável "Conhecimento" a partir de nove itens elaborados no questionário. Desta forma, observou-se no Quadro 2 as informações coletadas acerca do conhecimento (dados descritivos da variável) dos internos de medicina sobre o rastreio precoce, a dor e o manejo dos cuidados paliativos na oncologia.

Quando questionados sobre a diferença do diagnóstico precoce e o rastreio do câncer foi visto que boa parte dos 
internos atingiu o nível 3 e 4 (80,2\%); quando perguntados sobre a a segurança em reconhecer os sinais e sintomas do câncer e em indicar o melhor método para autoexame e o rastreio, alcançaram os níveis $3(63,4 \%)$ e $4(67,3 \%)$, respectivamente.

Notou-se que a maioria dos participantes reconheceu a diferença entre a dor nociceptiva e a dor neuropática, pois alcançaram os níveis 3 e 4 (80,2\%); porém, quando interrogados sobre a utilização de escalas para avaliação da dor, a maior parte afirmou não utilizar e/ou usar poucas vezes (56,5\% da amostra esteve nos níveis 0,1 e 2 da escala). Também, em relação a segurança em iniciar o manejo da analgesia da dor em um paciente oncológico, 51,5\% atingiram os níveis 3 e 4 . Entretanto, quando questionados sobre a necessidade de aprimorar seus conhecimentos sobre o manejo da dor, 96\% (soma dos níveis 2 e 3) reconheceu que necessitava deste aprimoramento.

Ainda, dentro da temática dos cuidados paliativos, quando indagados sobre a importância de um paciente oncológico em estado terminal morrer em casa junto com a sua família, foi visto que $82,2 \%$ concordaram parcialmente e/ou totalmente (níveis 3 e 4). No que concerne a estarem preparados para lidar com os cuidados terminais e com a morte de um paciente oncológico e, posteriormente, com a fase de luto dos familiares, a amostra mostrou-se dividida, apontando que 51,5\% dos estudantes atingiram níveis de 0 a 2 e a soma dos níveis 3 e 4 representam 48,5\%.

Fazendo uma avaliação geral sobre todas as perguntas que avaliaram a variável "conhecimento", constatou-se que a média de pontos foi de 24,8713 pontos, uma mediana de 26 pontos, com um desvio padrão de 4,60361. Além disso, o ponto máximo atingido foi de 34,00 pontos e o mínimo foi de 11,00 pontos.

Quadro 2: Estatísticas descritivas da variável "Conhecimento sobre o rastreio precoce, a dor e o manejo dos cuidados paliativos na oncologia." $(\mathrm{n}=101)$.

\begin{tabular}{|c|c|c|}
\hline Alternativas & Frequência & Porcentual \\
\hline Discordo totalmente & 6 & 5,9 \\
\hline Discordo Parcialmente & 6 & 5,9 \\
\hline Neutro & 8 & 7,9 \\
\hline Concordo Parcialmente & 29 & 28,7 \\
\hline Concordo Totalmente & 52 & 51,5 \\
\hline \multicolumn{3}{|c|}{ Você se sente seguro em reconhecer os sinais e sintomas do Câncer? } \\
\hline Alternativas & Frequência & Porcentual \\
\hline Discordo totalmente & 8 & 7,9 \\
\hline Discordo Parcialmente & 12 & 11,9 \\
\hline Neutro & 17 & 16,8 \\
\hline Concordo Parcialmente & 59 & 58,4 \\
\hline Concordo Totalmente & 5 & 5,0 \\
\hline total & 101 & 100,0 \\
\hline \multicolumn{3}{|c|}{ Você se sente seguro em indicar o melhor método para autoexame e rastreio do Câncer } \\
\hline Alternativas & Frequência & Porcentual \\
\hline Discordo Totalmente & 1 & 1,0 \\
\hline Discordo Parcialmente & 17 & 16,8 \\
\hline Neutro & 15 & 14,9 \\
\hline Concordo Parcialmente & 41 & 40,6 \\
\hline Concordo Totalmente & 27 & 26,7 \\
\hline \multicolumn{3}{|c|}{ Você sabe a diferença entre dor nociceptiva e neuropática? } \\
\hline Alternativas & Frequência & Porcentual \\
\hline Discordo Totalmente & 8 & 7,9 \\
\hline Discordo Parcialmente & 4 & 4,0 \\
\hline Neutro & 8 & 7,9 \\
\hline Concordo Parcialmente & 34 & 33,7 \\
\hline Concordo Totalmente & 47 & 46,5 \\
\hline \multirow{2}{*}{\multicolumn{3}{|c|}{$\begin{array}{l}\text { Durante a sua prática médica, você utiliza alguma escala para avaliação de dor? } \\
\text { Alternativas }\end{array}$}} \\
\hline Alternativas & & Porcentual \\
\hline
\end{tabular}




\begin{tabular}{|c|c|c|}
\hline Discordo Totalmente & 3 & 3,0 \\
\hline Discordo Parcialmente & 15 & 14,9 \\
\hline Neutro & 39 & 38,6 \\
\hline Concordo Parcialmente & 0 & 0,0 \\
\hline Concordo Totalmente & 44 & 43,6 \\
\hline \multicolumn{3}{|c|}{ Você acha necessário melhorar seu conhecimento no tratamento de pacientes com dor? } \\
\hline Alternativas & Frequência & Porcentual \\
\hline Discordo Totalmente & 2 & 2,0 \\
\hline Neutro & 2 & 2,0 \\
\hline Concordo Parcialmente & 37 & 36,6 \\
\hline Concordo Totalmente & 60 & 59,4 \\
\hline \multicolumn{3}{|c|}{$\begin{array}{c}\text { Caso você atenda um paciente oncológico com dor, você se sentiria seguro para iniciar o manejo da } \\
\text { analgesia? }\end{array}$} \\
\hline Alternativas & Frequência & Porcentual \\
\hline Discordo totalmente & 9 & 8,9 \\
\hline Discordo Parcialmente & 23 & 22,8 \\
\hline Neutro & 17 & 16,8 \\
\hline Concordo Parcialmente & 42 & 41,6 \\
\hline Concordo Totalmente & 10 & 9,9 \\
\hline \multicolumn{3}{|c|}{ Você acha importante um paciente oncológico em estado terminal morrer em casa junto com a sua família? } \\
\hline Alternativas & Frequência & Porcentual \\
\hline Discordo totalmente & 2 & 2,0 \\
\hline Discordo Parcialmente & 4 & 4,0 \\
\hline Neutro & 12 & 11,9 \\
\hline Concordo Parcialmente & 23 & 22,8 \\
\hline Concordo Totalmente & 60 & 59,4 \\
\hline \multicolumn{3}{|c|}{$\begin{array}{l}\text { Você acha que está preparado para lidar com os cuidados terminais e com a morte de um paciente oncológico } \\
\text { e posteriormente com a fase de luto dos familiares? }\end{array}$} \\
\hline Alternativas & Frequência & Porcentual \\
\hline Discordo totalmente & 8 & 7,9 \\
\hline Discordo Parcialmente & 20 & 19,8 \\
\hline Neutro & 24 & 23,8 \\
\hline Concordo Parcialmente & 40 & 39,6 \\
\hline Concordo Totalmente & 9 & 8,9 \\
\hline
\end{tabular}

Fonte: Dados da pesquisa (2021).

Quando perguntados sobre terem recebido, durante a graduação, informações suficientes para realizar o diagnóstico precoce e o rastreio de pacientes oncológicos de forma eficiente, a maioria estava nas escalas 3 e 4 (50,5\%). Essa situação se repetiu quando questionado sobre o recebimento de informações suficientes para realizar o manejo de pacientes com dor, apresentando a soma dos níveis 3 e $4(55,5 \%)$.

Entretanto, quanto ao acesso de informações suficientes, durante a graduação, sobre controle de sintomas mais comuns (dispneia, vômitos, obstipação, caquexia) em pacientes em cuidados paliativos, foi visto que 63,4\% estavam entre os níveis 0,1 e 2 . A mesma realidade se apresenta quando indagados sobre o manejo dos cuidados de pacientes em situação terminal com segurança, em que os níveis 0,1 e 2 totalizam 76,2\% da amostra.

$\mathrm{Na}$ mesma perspectiva de recebimento de informações durante a formação, indagou-se sobre as ferramentas de comunicação e a postura médica para “dar más notícias” aos pacientes e familiares, notando-se que $93 \%$ da amostra representam a soma dos graus 3 e 4.

Realizando uma avaliação global sobre todas as perguntas relacionadas com a variável "Oportunidade de Conhecimento", com pontuação variando entre 0 a 4 entre os respectivos graus, constatou-se que a média de pontos foi de 11,0990 pontos, uma mediana de 11,00 pontos, com um desvio padrão de 3,59584. Além disso, o ponto máximo atingido foi de 19,00 pontose o mínimo foi de 4 pontos. 
Quadro 3 - Estatística descritiva da variável "Oportunidade de receber o conhecimento rastreio precoce, a dor e o manejo dos cuidados paliativos na oncologia" $(\mathrm{n}=101)$.

\begin{tabular}{|c|c|c|}
\hline \multicolumn{3}{|c|}{$\begin{array}{l}\text { Você acredita que durante a graduação recebeu informação suficiente para realizar o diagnóstico precoce e o } \\
\text { rastreio de pacientes oncológicos de forma eficiente? }\end{array}$} \\
\hline Alternativas & Frequência & Porcentual \\
\hline Discordo Totalmente & 19 & 18,8 \\
\hline Discordo Parcialmente & 17 & 16,8 \\
\hline Neutro & 14 & 13,9 \\
\hline Concordo Parcialmente & 44 & 43,6 \\
\hline Concordo Totalmente & 7 & 6,9 \\
\hline \multicolumn{3}{|c|}{$\begin{array}{l}\text { Você acredita que durante a graduação recebeu informação suficiente para realizar o manejo de pacientes } \\
\text { com dor? }\end{array}$} \\
\hline Alternativas & Frequência & Porcentual \\
\hline Discordo Totalmente & 3 & 3,0 \\
\hline Discordo Parcialmente & 32 & 31,7 \\
\hline Neutro & 10 & 9,9 \\
\hline Concordo Parcialmente & 42 & 41,6 \\
\hline Concordo Totalmente & 14 & 13,9 \\
\hline \multicolumn{3}{|c|}{$\begin{array}{l}\text { Você acredita que durante a graduação recebeu informação suficiente sobre controle de sintomas mais } \\
\text { comuns (dispneia, vômitos, obstipação, caquexia) em pacientes em cuidados paliativos? }\end{array}$} \\
\hline Alternativas & Frequência & Porcentual \\
\hline Discordo Totalmente & 21 & 20,8 \\
\hline Discordo Parcialmente & 25 & 24,8 \\
\hline Neutro & 18 & 17,8 \\
\hline Concordo Parcialmente & 32 & 31,7 \\
\hline Concordo Totalmente & 5 & 5,0 \\
\hline Total & 101 & 100,0 \\
\hline \multicolumn{3}{|c|}{$\begin{array}{l}\text { Você aprendeu durante a graduação ferramentas de comunicação e postura médica para "dar más notícias" } \\
\text { aos pacientes e familiares? }\end{array}$} \\
\hline Alternativas & Frequência & Porcentual \\
\hline Discordo Totalmente & 2 & 2,0 \\
\hline Neutro & 5 & 5,0 \\
\hline Concordo Parcialmente & 37 & 36,6 \\
\hline Concordo Totalmente & 57 & 56,4 \\
\hline \multicolumn{3}{|c|}{$\begin{array}{l}\text { Você acredita que durante a graduação recebeu informação suficiente para realizar o manejo dos cuidado de } \\
\text { pacientes em situação terminal com segurança? }\end{array}$} \\
\hline Alternativas & Frequência & Porcentual \\
\hline Discordo Totalmente & 26 & 25,7 \\
\hline Discordo Parcialmente & 23 & 22,8 \\
\hline Neutro & 28 & 27,7 \\
\hline Concordo Parcialmente & 21 & 20,8 \\
\hline Concordo Totalmente & 3 & 3,0 \\
\hline
\end{tabular}

Fonte: Dados da pesquisa (2021).

O Quadro 4 representa a percepção da amostra quando questionada sobre a existência de uma disciplina específica de Oncologia no curso de medicina do UNIFIP. Foi constatado que o nível zero, que ilustra uma negativa quanto a pergunta, representa $87,1 \%$ dos entrevistados. E fazendo uma avaliação global, verificou-se que a média de pontos foi de 0,39 pontos, uma mediana de 0,00 pontos, com um desvio padrão de 0,871. Além disso, o ponto máximo atingido foi de 4 pontose o mínimo foi de 0 pontos. 
Quadro 4 - Estatística descritiva da pergunta "existência de disciplina Específica de Oncologia?” (n=101).

\begin{tabular}{|l|c|c|}
\multicolumn{2}{|c|}{ Existe na sua Faculdade uma disciplina específica de Oncologia? } \\
\hline \multicolumn{1}{|c|}{ Alternativa } & Frequência & Porcentual \\
\hline Discordo totalmente & 81 & 80,2 \\
\hline Discordo Parcialmente & 7 & 6,9 \\
\hline Neutro & 8 & 7,9 \\
\hline Concordo Parcialmente & 4 & 4,0 \\
\hline Concordo Totalmente & 1 & 1,0 \\
\hline Total & 101 & 100,0 \\
\hline
\end{tabular}

Fonte: Dados da pesquisa (2021).

No Quadro 5 é demonstrada a forma como foi aplicado o Teste de Normalidade de Shapiro-Wilk, a variável “Conhecimento" foi classificada como assimétrica $(\mathrm{E}=0,971 ; \mathrm{p}=0,020)$. Dado que H0 $>0,05$ significa que a amostra possui distribuição normal e que $\mathrm{H} 1<0,05$ significa distribuição assimétrica, verificamos pelo teste de Shapiro-Wilk que a variável "Conhecimento" possui distribuição assimétrica $(\mathrm{p}=0,020)$.

Além disso, apresenta os dados referentes à comparação de médias entre grupos no teste de Mann-Whitney. Sendo a variável "Conhecimento" uma soma dos escores de todos os itens dos questionários aplicados. Verificou-se haver diferenças no nível de segurança e conhecimento sobre oncologia entre os grupos do $5^{\circ}$ ano e do $6^{\circ}$ ano de internos de Medicina $(\mathrm{U}=$ 592,500; $\mathrm{p}<0,00$ ), em que os do $6^{\circ}$ ano mostraram-se mais seguros (Média $=67,05$ ) do que os do $5^{\circ}$ ano $($ Média $=43,21$ ) quanto ao rastreio precoce, a dor e o manejo dos cuidados paliativos na oncologia.

Quadro 5: Classificações e estatísticas do Testes de normalidade e de Mann-Whitney.

\begin{tabular}{|c|c|c|c|c|}
\hline \multirow{15}{*}{ Conhecimento } & Grupo Rodízio & $\mathbf{N}$ & Média em Ranks & Soma dos Ranks \\
\hline & $5^{\circ}$ Ano & 68 & 43,21 & 2938,50 \\
\hline & $6^{\circ}$ Ano & 33 & 67,05 & 2212,50 \\
\hline & Total & 101 & & \\
\hline & \multicolumn{4}{|c|}{ Estatísticas } \\
\hline & \multirow{3}{*}{\multicolumn{2}{|c|}{ Kolmogorov-Smirnov $\mathrm{a}^{\mathrm{a}^{1}}$}} & Estatística & 0,112 \\
\hline & & & $\mathrm{DF}$ & 101 \\
\hline & & & sig & 0,003 \\
\hline & \multirow{3}{*}{\multicolumn{2}{|c|}{ Shapiro-Wilk }} & Estatística & 0,967 \\
\hline & & & $\mathrm{DF}$ & 101 \\
\hline & & & sig & 0,012 \\
\hline & Mann-Whitney U & 592,500 & & \\
\hline & Wilcoxon W & 2938,500 & & \\
\hline & $\mathrm{Z}$ & $-3,846$ & & \\
\hline & P-valor (2 caudas) & ,000 & & \\
\hline
\end{tabular}

a. Variável de agrupamento: Grupo Rodízio $\quad a^{1}$. Lilliefors Significance Correction Fonte: Dados da pesquisa (2021).

\section{Discussão}

Nesta pesquisa foi observado que a faixa etária (23-26 anos) e o gênero feminino dos internos de medicina estão de acordo com os dados obtidos por Donizeti Alves Jr, Fonseca, Gutterres e Souza (2019), Orth et al. (2019), Sousa e Roriz (2021). Entretanto, a prevalência do gênero feminino não corrobora com os achados Sá, Sousa e Roriz (2021), pois seus resultados apontaram para uma maioria do gênero masculino.

Quanto ao estado civil, a maior parte dos participantes era solteira, achado análogo aos resultados de Teixeira, Costa, Mattos e Pimentel (2019). A respeito do ano do curso em os estudantes estão matriculados, os dados obtidos por essa pesquisa 
foram similares aos encontrados por Sá, Sousa e Roriz (2021), em que verificou uma prevalência dos alunos do $5^{\circ}$ ano, porém, apresentaram divergência em comparação com os estudos feitos por Foltran, Pizzol, Guimarães, Santana e Fernandes (2015), os quais encontraram um maior número internos no $6^{\circ}$ ano.

Quanto ao conhecimento de estudantes de medicina sobre o rastreio precoce, a dor e o manejo dos cuidados paliativos na oncologia, Buranello, Meirelles, Walsh, Pereira, e Castro (2014), ressaltou a importância da instauração de estratégias baseadas em evidências para a prevenção, detecção precoce e início do tratamento o quanto antes, para um melhor prognóstico do paciente. Dessa forma, observou-se que tal achado se assemelhou aos encontrados neste trabalho, uma vez que boa parte dos estudantes apresentou um nível de conhecimento favorável sobre o rastreio e o diagnóstico precoce do câncer, além da indicação do melhor seguimento clínico para o decorrer do tratamento desse paciente.

Entretanto, pesquisas com grupo amostral semelhante apontaram para uma realidade controversa. de Oliveira, Faleiro e Alves (2019) evidenciou que a maior parte dos entrevistados reconheceram não ter conhecimento suficiente sobre a prevenção e detecção do câncer de forma precoce.

No que concerne à importância do estudante da área de saúde saber diferenciar dor nociceptiva e dor neuropática, os estudos desenvolvidos por Sousa e Roriz (2021) apontam que tal distinção é crucial à decisão pelo melhor tratamento destinado ao paciente, sobretudo quanto o mesmo se encontra em estado terminal, mas contrariamente, os citados autores encontraram valores que demonstravam para um cenário preocupante, já que seus resultados indicaram que a amostra desconhecia a diferença de tais conceitos. Por conseguinte, os achados deste estudo sinalizaram um cenário favorável, pois foi observado que a maior parte dos internos reconheceu as diferenças entre ambas.

Ademais, no tocante a utilização de escalas para mensurar a dor, sabe-se que é fundamental na prática clínica, pois, além de manter avaliação constate e periódica, possibilita a escolha de um tratamento cada vez mais humanizado e individualizado para cada paciente. (Monfrim, Saraiva, Moraes \& Viegas, 2015). Entre os participantes desta pesquisa em Patos-PB, 56,6\% afirmaram não utilizar e/ou usar poucas vezes escalas para a avaliação da dor, fato que pode ter consequências negativas sobre o bem estar do paciente. Outros estudos, contudo, indicaram que a maioria dos participantes conhecia e utilizava escalas para a dor na realidade de seus pacientes (Pinheiro, 2010; Sousa \& Roriz, 2021).

Com relação ao início do manejo da analgesia da dor em um paciente oncológico, Hennemann-Krause e Sredni (2016) ressaltaram que a utilização de drogas (opioides, antidepressivos, Gabapentinoides) comumente administradas em dor neuropática, de maneira imprecisa e errônea, pode gerar diversos efeitos adversos, como: sonolência, tonteiras, hipotensão ortostática, sedação e depressão respiratória. Entende-se a importância e a dificuldade em se obter segurança para tal prática.

Os participantes desta pesquisa apresentaram-se divididos, pois, apenas 52,8\% dos entrevistados afirmaram ter segurança em inciar esse tratamento. Tal fato pode ser sustentado pelo estudo de Sousa e Roriz (2021), que afirmaram que uma parcela alta dos pesquisados relataram preocupação com o risco de causar uma depressão respiratória, comum em caso de excesso de dose (overdose) e a dependência química pelo uso de opioide.

Ademais, um ponto importante desta abordagem foi que e 95,3\% dos estudantes entrevistados reconheceram que necessitavam aprimorar seus conhecimentos sobre o manejo da dor nos pacientes oncológicos. Segundo Sousa e Roriz (2021) a grande maioria das faculdades do nordeste, inclusive o UNIFIP (local da pesquisa), não dispõe de um componente teórico especificamente voltado para esse ponto.

Igualmente, foi visto que $82,1 \%$ dos internos reconheceram a relevância de um paciente oncológico em estado terminal morrer em casa junto com a sua família. Fato este que é sustentado por Frizzo, Bertolini, Caron, Steffani e Bonamigo (2013), os quais observaram que 95,9\% dos entrevistados também reconhecem tal importância.

Ainda dentro da temática dos cuidados paliativos, constatou-se que 51,9\% desses estudantes disseram estar preparados para lidar com os cuidados terminais e com a morte de um paciente oncológico e, posteriormente, com a fase de 
luto dos familiares. Constatação semelhante foi apontada por Frizzo, Bertolini, Caron, Steffani e Bonamigo (2013), já que $58,9 \%$ dos acadêmicos de sua amostra responderam estar apta. Por conseguinte, Sousa e Roriz (2021) destacaram que $86 \%$ dos internos abordados apontaram aptidão.

Com relação à oportunidade em se obter conhecimento, dados análogos a esta pesquisa foram identificados. Oliveira, Faleiro e Alves (2019) observaram que 96,3\% dos alunos receberam informação em alguma parte do curso de medicina sobre as diretrizes para para rastreamento do câncer de colo uterino.

Quanto à quantidade de informação recebida pelos acadêmicos para o manejo de pacientes com dor, o resultado encontrado nesse estudo apontou para uma realidade dividida. Entretanto, outras pesquisas como Pinheiro (2010), Dalpai et al., (2017); Braide, Leal e Souza (2019), Sousa e Roriz (2021) identificaram resultados discordantes aos deste estudo, já que a maior parcela do estudantes afirmaram não ter recebido informações suficientes.

Braide, Leal e Souza (2019) ressaltaram a existência de lacunas na preparação dos futuros médicos. Os autores sinalizaram para o déficit de conhecimentos sobre controle de sintomas mais comuns (dispneia, vômitos, obstipação, caquexia) em pacientes em cuidados paliativos e sobre o manejo dos pacientes em situação terminal. Esta também foi a realidade deste estudo.

Importante frisar, contudo, a constatação de que os estudantes afirmaram manejar oportunamente a comunicação e a transmissão de informação, sobretudo para "dar más notícias" aos pacientes e familiares. Sinergia com os resultados encontrados duas outras pesquisas; Braide, Leal e Souza (2019); Sousa e Roriz (2021). Estes achados podem indicar a competência dos estudantes entrevistados quanto à postura médica, bem como a capacidade de transmtir informações difíceis. Afinal, diante da experiência com situações difíceis e de perda, é imprescindível o suporte emocional e social Suárez, Sousa e Caldas (2021).

Ressalta-se que no curso de medicina do UNIFIP, o eixo Humanidads Médicas tem tido um papel importante na formação, especialmente quanto a este processo de ensinagem, pois está na matriz curricular o tema comunicação clínica: a relação terapêutica, situações dolorosas, negociando com as crenças e as emoções do paciente.

Calil e Prado (2009) mostraram que o perfil epidemiológico brasileiro aponta o Câncer como uma das principais causas de mortalidade e evidenciaram a importância de que, em sua formação acadêmica, um profissional de saúde deve desenvolver habilidades que o tornem capaz de reconhecer e intervir sobre os problemas/situações de saúde-doença mais prevalentes no perfil epidemiológico da sua região de atuação. Além disso, Silvestrini, Scherrer e Moreira (2012), em um estudo envolvendo 110 faculdades de medicina no Brasil, dissertaram que na maioria dessas finstituições, a disciplina de oncologia não é ofertada em sua grade curricular, o que não permite que o aluno receba a informação de uma maneira mais aprofundada e especializada sobre o agravo. Neste estudo, a grande maioria dos entrevistados atestou não possuir em sua grade curricular a disciplina de oncologia.

Ainda sobre a disponibilidade de uma disciplina específica de oncologia na faculdade de medicina, pesquisa realizada por Silvestrini, Scherrer e Moreira (2012), analisando uma amostra de 100 faculdades de Medicina no Brasil, observaram que $70 \%$ delas não possuíam um componente curricular específico em sua matriz curricular. E, ao observar as Diretizes Curriculares Nacionais (DCNs) para o curso, não há a obrigatorietadade do ensino de oncologia, nem básica e nem avançada (Brasil, 2014).

Por fim, constatou-se que, ao comparar estudantes do $5^{\circ}$ e $6^{a}$ anos quanto ao conhecimento sobre rastreio precoce, dor e o manejo dos cuidados paliativos na oncologia, os internos do $6^{\circ}$ ano apresentaram melhores resultados, fato que se justifica pela expectativa de que haja uma evolução gradativa com o decorrer do curso, por parte desses estudantes, com relação ao seu raciocício e a sua percepção clínica sobre os pacientes Frizzo, Bertolini, Caron, Steffani e Bonamigo (2013).

Ao fim dessas análises, constatou-se a dificuldade para a construção dessa pesquisa visto que não havia estudos 
suficientes e atualizados realizados sobre esse tema. Além disso, a maioria dos estudos encontrados não era direcionada a escola médica e sim, de forma generalista entre os demais profissionais de saúde.

\section{Conclusão}

Apesar da necessidade de discutir e ter acesso à informação inerente às doenças crônicas não transmissíveis, entre elas o câncer, condição clínica com incidência e prevalência crescente, observa-se que na instituição de pesquisa não há um componente específico de Oncologia, o que revela um cenário preocupante diante da nova realidade da prática médica brasileira.

Apesar disto, ao analisar os resultados referentes ao conhecimento dos acadêmicos do quinto e do sexto ano de medicina acerca do diagnóstico precoce e rastreio, a dor e o manejo dos cuidados paliativos, foi evidenciado a existência de favorável nível de conhecimento clínico, porém, ainda há a necessidade de aprimorá-lo.

Além disso, fica evidente a necessidade de incentivar a produção científica médica acerca da oncologia, sobretudo sobre o diagnóstico precoce e rastreio, a dor e o manejo dos cuidados paliativos, durante toda a vida médica, tendo em conta a nova realidade epimiológica do Brasil.

Espera-se que os dados agrupados e debatidos neste estudo sejam capazes de colaborar para que os pacientes oncológicos possam receber um atendimento cada vez mais humanizado e individualizado, visando uma melhoria na sua qualidade de vida desde o início do seu tratamento até o fim de sua vida. Ademais, presume-se a necessidade de uma reflexão acerca de um novo modelo de currículo médico, ou seja, fica clara a necessidade de uma reforma na matriz curricular das escolas médicas, com a incorporação de Oncologia no currículo.

\section{Referências}

Donizeti Alves Jr, V. D. A. J. A., Fonseca, S. R., Gutterres, D. B., \& Souza, M. C. A. de (2019). Cuidados paliativos: conhecimento de estudantes de graduação em enfermagem e em medicina. Revista De Saúde, 10(2), 07-11. https://doi.org/10.21727/rs.v10i2.1744

Batista, D. R. R., Mattos, M. De., \& Silva, S. F. da (2015). Convivendo com o câncer: do diagnóstico ao tratamento. Revista de Enfermagem da UFSM, 5(3), 499-510. http://dx.doi.org/10.5902/2179769215709

Braide, C. S. L., Leal, P. da C., \& Souza, M. H. S. L. De (2019). Avaliação do grau de conhecimento sobre cuidados paliativos e dor dos estudantes de medicina em uma faculdade particular de São Luís/MA. Revista de Investigação Biomédica, 10(3), 207-218. http://dx.doi.org/10.24863/rib.v10i3.314

Buranello, C. M., Meirelles, M. C. C. C., de Walsh, I. A. P., Pereira, G. A. P., \& Castro, S. S. (2018). Prática de exames de rastreio para câncer de mama e fatores associados - Inquérito de Saúde da Mulher em Uberaba MG, Brasil, 2014. Ciência \& Saúde Coletiva,, 23(8), 2661-2670. https://doi.org/10.1590/141381232018238.14762016

Calil, A. M., \& Prado, C. (2010). Ensino de oncologia na formação do enfermeiro. Rev. Bras. Enferm, 63(4), 671-674. https://doi.org/10.1590/S003471672010000400026

Dalpai , D., Mendes , F. F., Asmar, J. A. V. N., Carvalho, P. L., Loro, F. L., \& Branco, A. (2017). Dor e cuidados paliativos: o conhecimento dos estudantes de medicina e as lacunas da graduação. Rev. dor, 18(4), 307-310. https://doi.org/10.5935/1806-0013.20170120

Dantas, B. M. S., Portugal, G. A., Silva, C. S., Galvão, L. R., Galvão, C. R., Santos, Ê. M. M., da Silva, C. A. L., \& Coelho, J. M. F. (2020). O estudo da oncologia nos cursos de graduação em enfermagem em um Estado do Nordeste Brasileiro. Brazilian Journal of Health Review, 3(4), 10664-10676. http://dx.doi.org/10.34119/bjhrv3n4-276

Frizzo, K., Bertolini, G., Caron, R., Steffani, J. A., \& Bonamigo, E. L. (2013). Percepção dos acadêmicos de medicina sobre cuidados paliativos de pacientes oncológicos terminais. Revista Bioethikos, 7(4), 367-375.

Foltran, C., Pizzol, L. R., Guimarães, M. L., de Santana, C., \& Fernandes, M. R. (2015). Avaliação do conhecimento na requisição correta dos exames de imagem dos internos de 5o e 60 ano da Faculdade de Medicina da Universidade de Mogi das Cruzes, SP. Rev Med (São Paulo), 94(2), 126-134. http://dx.doi.org/10.11606/issn.1679-9836.v.94i2p75-80

Fonseca, A., \& Geovanini, F. (2013). Cuidados Paliativos na Formação do Profissional da Área de Saúde. Revista Brasileira de Educação Médica, 37(1), 120125. https://doi.org/10.1590/S0100-55022013000100017

Hennemann-Krause, L., \& Sredni, S. (2016). Systemic drug therapy for neuropathic pain. Rev Dor. São Paulo, 17(1), 91-94. https://doi.org/10.5935/18060013.20160057 
Kameo, S. Y., Barbosa-Lima, R., Ramos, M. J. O., Fonseca, T. V., Vassilievitch, A. C., Costa, J. dos S., Santos, J. C. de O., Santos, D. K. da C., Amorim, B. F., Marinho, P. M. L., Sawada, N. O., \& Silva, G. M. (2021). Perfil clínico-epidemiológico de mulheres em tratamento oncológico de carcinoma ductal invasivo da mama. Research, Society and Development, 10(1), e39110111836. https://doi.org/10.33448/rsd-v10i1.11836

Luz, K. R. D., Vargas, M. A. D. O., Rosa, L. M. D., \& Schmitt, P. H. (2016). Enfermeiros na atenção oncológica: conhecimento na prática do cuidado. Rev. enferm. UFPE on line, 10(9), 3369-3376. https://doi.org/10.5205/1981-8963-v10i9a11418p3369-3376-2016

Ministério da Educação (BR). (2014). Resolução no 3, de 20 de Junho de 2014. Diretrizes curriculares nacionais do curso de graduação em medicina. Brasília: Ministério da Educação.

Monfrim, X. M., Saraiva, L. D. A., De Moraes, C. L., \& Viegas, A. D. C. (2015). Escala de avaliação da dor: percepção dos enfermeiros em uma Unidade de Terapia Intensiva Neonatal. Revista de Enfermagem da UFSM, 5(1), 12-22. https://doi.org/10.5902/2179769215049

de Oliveira, T. S., Faleiro, N. Q., \& Alves, R. R. F. (2019). Conhecimento dos estudantes de medicina a respeito da triagem citológica do câncer de colo uterino. Revista EVS - Revista de Ciências Ambientais e Saúde, 46, 44-52. https://doi.org/10.18224/evs.v46i1.6443

Orth, L. C., Haragushiku, E. Y., Freitas, I. C. S., Hintz, M. C., Marcon, C. E. M., \& Teixeira, J. F. (2019). Conhecimento do Acadêmico de Medicina sobre Cuidados Paliativos. Revista Brasileira de Educação Médica, 43(1), 286-295. https://doi.org/10.1590/19815271v43suplemento1-20190039

Peirano, G., Bertolino, M., Rodriguez, M., Bunge, S., Armesto, A., \& Dran, G. (2019). Interconsultas de cuidados paliativos para pacientes con cáncer en un hospital general. Características y oportunidad. Medicina (B. Aires), 79(5), 337-344.

Pinheiro, T. R. S. P. (2010). Avaliação do grau de conhecimento sobre cuidados paliativos e dor dos estudantes de medicina do quinto e sexto anos. $O$ Mundo da Saúde, São Paulo, 34(3), 320-326. https://doi.org/10.15343/0104-7809.20103320326

Sá, A. H. M., Roriz, M. I. R. C., \& de Sousa, M. N. A. (2021). Avaliação do conhecimento de internos de medicina sobre odiagnóstico e tratamento do acidente vascular encefálico. Brazilian Journal of Development, 7(2), 20515-20526. https://doi.org/10.34117/bjdv7n2-613

Santos, A. P. B. Dos., Santos, R. N. O., Farias, Q. S. dos S., Santos, J. L. B. S., Martins, M. de C. V., \& Gallotti, F. C. M. (2021). Capacitação profissional e sua articulação na assistência de enfermagem à criança com câncer. Research, Society and Development, 10(6), e4710615475. https://doi.org/10.33448/rsdv10i6.15475

Silva, A. E., Guimarães, M. A. M., Carvalho, R. C., Carvalho, T. V., Ribeiro, S. A., \& Martins, M. R. (2021). Cuidados paliativos: definição e estratégias utilizadas na prática médica. Research, Society and Development, 10(1), e18810111585. https://doi.org/10.33448/rsd-v10i1.11585

Silva, D. A. D., Silva, K. A., \& Porfírio, R. B. M. (2020). Câncer: Repercussões psicológicas relacionadas com a trajetória da doença oncológica. Revista Científica Multidisciplinar Núcleo do Conhecimento, 2(1), 31-43.

Silva, A. L. M. Da., Silva, W. T. da., Teixeira, E. B., Khayat, A. S., Rodrigues, T. V. P., \& Khayat, B. C. M. (2021). O papel do agente comunitário de saúde na disseminação de informações acerca dos principais cânceres de interesse na atenção básica. Research, Society and Development, $10(1)$, e24810111556. https://doi.org/10.33448/rsd-v10i1.11556

Silveira, P. J. Da., Costa, A. E. K. Da., Lohmann, P. M., \& Lavall, E. (2020). Revisão integrativa: cuidados paliativos em pacientes oncológicos. Research, Society and Development, 9(2), e144922136. https://doi.org/10.33448/rsd-v9i2.2136

Silvestrini, A. A., Scherrer, L. R., \& Moreira, W. B. (2012). O ensino de oncologia na graduação: panorama brasileiro. Revista Brasileira de Oncologia Clínica, 8(29), 125-129.

Soares, A. S., Shitsuka, D. M., Parreira, F. J., \& Shitsuka, R. (2018). Metodologia da pesquisa científica (1st ed.). UFSM, NTE.

Sousa, M. N. A., \& Roriz, M. I. R. C. (2021). Avaliação do conhecimento de estudantes de medicina sobre dor em cuidados paliativos. Brazilian Journal of Development, 4(1), 3425-3436. https://doi.org/10.34119/bjhrv4n1-275

Suárez, L. de A. B., Sousa, M. N. A. de., \& Caldas, M. T. (2021). A dor sem nome: análise da experiência de mães que perderam filhos. Research, Society and Development, 10(1), e39010111824. https://doi.org/10.33448/rsd-v10i1.11824

Teixeira, L. D. A. C., Costa, R. A., de Mattos, R. M. P. R., \& Pimentel, D. (2021). Saúde mental dos estudantes de Medicina do Brasil durante a pandemia da coronavirus disease 2019. J Bras Psiquiatr, 70(1), 21-29. https://doi.org/10.1590/0047-2085000000315

Theobald, M. R., Santos, M. L. de M. dos, Andrade, S. M. O. de, De-Carli, A. D. (2016). Percepções do paciente oncológico sobre o cuidado. Physis: Revista de Saúde Coletiva, 26(4), 1249-1269. http://dx.doi.org/10.1590/s0103-73312016000400010. 